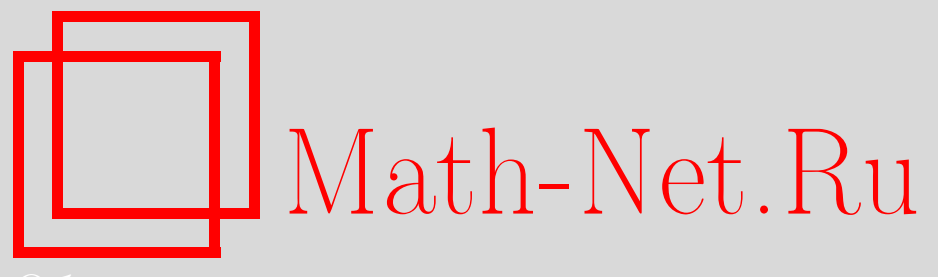

С. Гонсалес, В. Т. Марков, К. Мартинес, А. А. Нечаев, И. Ф. Руа, Неассоциативные кольца Галуа, Дискрет. матем., 2002, том 14, выпуск 4, 117-132

DOI: https://doi.org/10.4213/dm267

Использование Общероссийского математического портала Math-Net.Ru подразумевает, что вы прочитали и согласны с пользовательским соглашением http: //www . mathnet.ru/rus/agreement

Параметры загрузки:

IP : 52.23 .180 .231

26 апреля 2023 г., 18:28:39

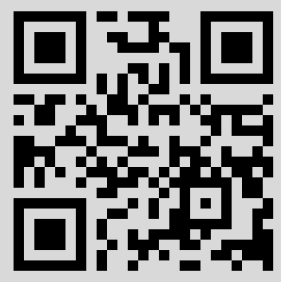


УдК 512.4

\title{
Неассоциативные кольца Галуа
}

( 2002 г. С. Гонсалес, В. Т. Марков, К. Мартинес, А. А. Нечаев, И. Ф. Руа

\begin{abstract}
Вводится и изучается понятие обобщенного кольца Галуа, то есть, кольца, удовлетворяющего всем аксиомам кольца Галуа, кроме аксиомы ассоциативности. На общий случай переносятся такие основные результаты, известные для ассоциативных колец Галуа, как описание решетки идеалов, мощности и характеристики. Для обобщенных колец Галуа также доказывается теорема существования. Тем не менее, теорема единственности, известная в ассоциативном случае, для обобщенных колец Галуа не верна.

Работа частично поддержана грантом FEDER (IFD-97-0556), грантом MCYT (PB-PGI99-04), грантом FICYT (PB-EXPO1-33).
\end{abstract}

\section{1. Введение}

Основы теории колец Галуа заложены в работе [8]. Эта теория была развита в работах [6], [15] и [12]. Позднее, в [14] изучались приложения этих колец к теории кодирования (представление нелинейных кодов над конечными полями как линейных над КГ) и криптографии [9]) (псевдослучайные последовательности, основанные на линейных рекуррентных последовательностях над кольцами Галуа). Развитие теории неассоциативных колец Галуа представляется интересным, главным образом с точки зрения возможности новых приложений в этих областях. Мы представляем неассоциативное обобщение понятия кольца Галуа.

Во втором разделе статьи рассматриваются ассоциативные кольца Галуа и конечные полутела, напоминаются определения и основные свойства. Третий раздел посвящен определению и основным свойствам обобщённых колец Галуа. Приведены примеры обобщенных колец Галуа, основанные на некоторых специфических полутелах. В четвертом разделе исследуются обобщенные кольца Галуа с ассоциативными степенями. Наконец, в последнем разделе доказана теорема существования для важного класса обобщенных колец Галуа (теорема о поднятии полутела) и обсуждается проблема единственности обобщенного кольца Галуа.

Ниже, если не оговорено противное, слово кольцо означает неассоциативное кольцо. Для любого кольца $S$, через $\Delta(S)$ обозначается множество всех делителей нуля (односторонних или двусторонних), включая 0 . 


\section{2. Кольца Галуа и полутела}

Определение 1. Конечное ассощиативное кольцо $S$ с единицей $e$ называется кольцом Галуа, если множество всех его делителей нуля $\Delta(S)$ равно $p S$ для некоторого натурального числа $p$.

Определение 2. Характеристикой char $S$ конечного кольца $S$ назовем экспоненту его аддитивной групты $(S,+)$. Если $S-$ кольцо с единицей $e$, то char $S=\operatorname{ord}_{+} e-$ аддитивный порядок элемента $е$ групшы $(S,+)$.

Если $S$ - кольцо Галуа, то оно коммутативно, $\Delta(S)=p S$ для некоторого простого числа $p$ и характеристика кольца $S$ равна $p^{n}$ для некоторого натурального числа $n$. Более того, $p S$ - нильрадикал кольца $S$, а его верхний фактор $\bar{S}=S / p S$ - конечное поле из $q=p^{r}$ элементов для некоторого $r \in \mathbf{N}$. В этом случае, число элементов кольца Галуа равно $q^{n}$. Для любого простого числа $p$ и любых $n, r \in \mathbf{N}$ существует единственное, с точностью до изоморфизма, кольцо Галуа $S$ мощности $q^{n}, q=p^{r}$, имеющее характеристику $p^{n}$. Оно обычно обозначается $G R\left(q^{n}, p^{n}\right)[11,12,15]$. Простейшими примерами колец Галуа являются конечные поля $G F(q)=G R\left(q^{1}, p^{1}\right), q=p^{r}$, и кольца вычетов кольца целых чисел $Z_{p^{n}}=G R\left(p^{n}, p^{n}\right)$ для любого простого числа $p$ и любого $n \in \mathbf{N}$.

Верхний фактор $\bar{S}=S / p S$ (конечное поле, строение которого хорошо известно) играет важную роль в исследовании ассоциативных колец Галуа. Многие свойства конечных полей (например, строение групты автоморфизмов или решетки подструктур) могут быть подняты на кольца Галуа. Именно, группа $\operatorname{Aut}(S)$ кольца Галуа $S=G R\left(q^{n}, p^{n}\right), q=p^{r},-$ циклическая группа порядка $r$, и для любого $t$, делящего $r$, существует единственное подкольцо $R=G R\left(\left(p^{t}\right)^{n}, p^{n}\right)$ кольца $S$ (см. [14]). Для любого кольца Галуа $S=G F\left(q^{n}, p^{n}\right)$ и для любого $d \in \mathbf{N}$ существует расширение $T=G R\left(\left(q^{d}\right)^{n}, p^{n}\right)$ кольца $S$ (см. [12]). Основными инструментами в процессе поднятия свойств с верхнего фактора на все кольцо служат неприводимые многочлены над конечными полями и соответствующие им многочлены над кольцами Галуа.

Мы увидим, что в новом контексте (при отсутствии ассоциативности) появляется более широкий спектр возможностей в изучении и развитии теории колец Галуа. Главная причина состоит в том, что обобщённое кольцо Галуа $S$ в качестве верхнего фактора $\bar{S}=S / p S$ имеет конечное полутело (иногда называемое в литературе (неассоциативным) кольцом с делением).

Определение 3. Конечное кольцо $D$ называется полутелом, если множество $D \backslash\{0\}$ замкнуто относительно умножения и является лупой. Это означает, что существует элемент $e \in D$ такой, что $e x=x e=x$ для любого элемента $x \in D$ и для произвольной пары элементов $a, b$ кольца $D, a \neq 0$, каждое из уравнений $a x=b$ и $x a=b$ имеет в $D$ единственное решение.

Первыми примерами полутел являются, конечно, поля. Напомним некоторые главные свойства конечных полутел (см., например, [7]).

Определение 4. Пусть $R$ - неассоциативное кольцо. Множество

$$
Z(R)=\{a \in R \mid \forall b, c \in R:(a b) c=a(b c)=b(a c), a b=b a\} .
$$

называется центром кольца $R$. 
Предложение 1. Пусть $D$ - конечное полутело. Тогда

(1) D имеет характеристику р для некоторого простого числа р;

(2) центр $Z(D)$ полутела $D-$ конечное поле из $p^{c}$ элементов, для некоторого $c \in \mathbf{N}$;

(3) $D$ - алгебра над полем $Z(D)$, содержащая $p^{c d}$ элементов, для некоторого $d \in \mathbf{N}$. Eсли $D$ не ассочиативно, то $d \geqslant 3$.

Как было отмечено выше, конечные поля являются простейшими примерами полутел. В этом случае центр полутела совпадает со всем кольцом и $d=1$. Теперь мы приведем некоторые примеры неассоциативных конечных полутел.

Пример 1 ([4]). Рассмотрим конечное поле $K$ из $q=p^{c e}$ элементов, где $p$ - нечетное простое число и $e \geqslant 2$. Пусть $g$ - такой элемент поля $K$, что многочлен $x^{2}-g$ неприводим в $K[x]$ (например, в качестве $g$ можно взять образующий элемент мультипликативной циклической группы $K^{*}$ ). Пусть $\sigma$ - нетождественный автоморфизм поля $K$, причем его поле инвариантов

$$
F=\{x \in K \mid \sigma(x)=x\}
$$

изоморфно $G F\left(p^{c}\right)$. Определим на множестве $S=K \times K$ операцию сложения покоординатно, а новую операцию умножения $*$ следующим образом:

$$
\forall a, b, c, d \in K:(a, b) *(c, d)=(a c+g \sigma(b) \sigma(d), a d+b c) .
$$

Тогда $(S,+, *)$ - неассоциативное конечное полутело из $q^{2}$ элементов, которое мы будем называть коммутативным полутелом Диксона. Его единицей является пара $(1,0)$.

Центр кольца $S$ есть, как нетрудно проверить,

$$
Z(S)=\{(a, 0): a \in K\}=G F(q) .
$$

Пример 2 ([2]). Рассмотрим конечное поле $F$ из $q=p^{c}>2$ элементов. Пусть $K=G F\left(q^{d}\right)$ - расширение поля $F$ степени $d \geqslant 3, c$ - элемент поля $K$ такой, что ord $c \nmid\left(q^{d}-1\right) /(q-1)$ (например, образующий элемент группы $\left.K^{*}\right)$. Пусть $\sigma \neq i d$ и $\tau \neq i d-$ два автоморфизма поля $K$, пересечение полей инвариантов которых совпадает с

$$
F=\{a \in K \mid \sigma(a)=\tau(a)=a\} .
$$

Определим новое произведение элементов множества $K$, полагая

$$
\forall x, y \in K: x \cdot y=x y-c \tau(x) \sigma(y) .
$$

Линейные операторы $R_{1}$ и $L_{1}$ на ${ }_{F} K$, задаваемые равенствами

$$
\forall x \in K: R_{1}(x)=x \cdot 1=x-c \tau(x), L_{1}(x)=1 \cdot x=x-c \sigma(x),
$$

являются невырожденными. Следовательно, можно рассмотреть обратные операторы $R_{1}^{-1}$ и $L_{1}^{-1}$. Теперь положим

$$
\forall x, y \in K: x * y=\left(R_{1}^{-1}(x)\right) \cdot\left(L_{1}^{-1}(y)\right) .
$$

Тогда $(K,+, *)$ - конечное полутело, называемое обобщённым скрученным полем. Оно неассоциативно, если $\sigma \neq \tau$, и в этом случае его единица - элемент $(1-c)$. 
Приведенные выше конструкции дают примеры различных полутел, мощность которых - фиксированная степень простого числа. Например, существует 24 попарно не изоморфных полутела мощности 16 и более чем 2000 (попарно не изоморфных) полутел мощности 32 (см. [3, 7]). Болышое число колец, которые существуют, и, тем самым, могут быть использованы в приложениях, - одна из главных мотивировок для разработки теории неассоциативных колец Галуа.

\section{3. Обобщенные кольца Галуа}

Здесь мы вводим понятие обобщенного кольца Галуа. При нашем определении (в котором отброшено требование ассоциативности) большинство основных свойств ассоциативных колец Галуа сохраняются.

Определение 5. Конечное кольцо $S$ с единищей $e$ называется обобщенным кольцом Галуа, если для некоторого натурального числа $\lambda$ выполняется равенство

$$
\Delta(S)=\lambda S
$$

Ясно, что каждое классическое кольцо Галуа $S=G R\left(q^{n}, p^{n}\right)$ есть обобщенное кольцо Галуа с $\Delta(S)=p S$. Любое конечное полутело $D$ характеристики $p$ также есть обобщенное кольцо Галуа. В этом случае $\Delta(D)=\{0\}=p D$.

Предложение 2. Пусть $S$-конечное кольчо с единицей е такое, что $\Delta(S)$ - двусторонний идеал кольча $S$. Тогда $\Delta(S)$ - множество всех двусторонних делителей нуля, включая 0, и верхний фактор $S / \Delta(S)$ - полутело.

Доказательство. Пусть $x \in \Delta(S) \backslash\{0\}$. Если $x$ не является двусторонним делителем нуля, предположим, что он - левый, но не правый делитель нуля. Тогда существует элемент $y \in S$ такой, что $y x=e$, поскольку $S$ - конечное кольцо. Так как $\Delta(S)$ - двусторонний идеал кольца $S$, справедливо соотношение $e=y x \in \Delta(S)$, получаем противоречие. Таким образом, $\Delta(S)$ - множество всех двусторонних делителей нуля кольца $S$. Следовательно, для любого элемента $a \in S \backslash \Delta(S)$ мощность каждого из множеств $a S$ и $S a$ равна мощности множества $S$, поэтому $a S=S a=S$ и тем более $\bar{a} \bar{S}=\bar{S} \bar{a}$, откуда и следует, что $\bar{S}=S / \Delta(S)$ - полутело.

Для любого обобщенного кольца Галуа $S$ мы будем обозначать его верхний фактор $S / \Delta(S)$ через $\bar{S}$ и через $\bar{a}$ будем обозначать образ произвольного элемента $a \in S$ в верхнем факторе $\bar{S}$ при каноническом эпиморфизме $S$ на $\bar{S}$.

Предложение 3. Пусть $S$ - обобщенное кольчо Галуа. Тогда наименьшее натуральное число $\lambda \in \mathbf{N}$, удовлетворяючее (4), является простым числом $р$ и верхний фактор $\bar{S}=S / \Delta(S)=S / p S-$ конечное полутело характеристики р. Более того, существует натуральное число $n \in \mathbf{N}$ такое, что характеристика $S$ равна $p^{n}$.

Доказательство. Если наименьшее натуральное число $\lambda \in \mathbf{N}$, удовлетворяющее (4), не является простым числом, то можно выбрать натуральные числа $\lambda_{1}, \lambda_{2}>1$ такие, что $\lambda_{1} \lambda_{2}=\lambda$. Если $\lambda_{1} e$ (соответственно, $\left.\lambda_{2} e\right)$ является делителем нуля, то, поскольку $\Delta(S)-$ двусторонний идеал кольца $S$, справедливы соотношения

$$
\lambda_{i} S=\lambda_{i}(e S)=\left(\lambda_{i} e\right) S \subseteq \Delta(S)=\lambda S \subseteq \lambda_{i} S,
$$


и $\lambda_{i} S=\Delta(S)$, что противоречит определению $\lambda$. Следовательно, в $\bar{S}=S / \Delta(S)$ получаем, что

$$
\overline{\lambda_{1}} \bar{e} \neq \overline{0}, \quad \overline{\lambda_{2}} \bar{e} \neq \overline{0}
$$

Так как $\bar{S}-$ полутело,

$$
\overline{0} \neq\left(\overline{\lambda_{1} e}\right)\left(\overline{\lambda_{2} e}\right)=\overline{\lambda e} .
$$

Поэтому $\lambda e \notin \Delta(S)=\lambda S$, что опять противоречит определению $\lambda$. Таким образом, $\lambda-$ простое число $p$ и $\bar{S}=S / \Delta(S)=S / p S$ - конечное полутело характеристики $p$.

Если $h$ - характеристика кольца $S$, то $h a=0$ для любого элемента $a \in S$. Поэтому $h \bar{a}=0$ для любого элемента $\bar{a} \in \bar{S}$, и характеристика $p$ полутела $\bar{S}$ делит $h$. Если $h=p^{n} l$, причем $\operatorname{gcd}(p, l)=1$, то существуют натуральные числа $r, s$ такие, что $1=p^{n} s+l t$. Теперь если $l>1$, то $p^{n} e \neq 0$, и ясно, что из равенства $\left(p^{n} e\right)(l e)=h e=0$ следует, что le $\in \Delta(S)=p S$. Таким образом, существует элемент $a \in S$ такой, что $l e=p a$. В этом случае

$$
e=1 e=p^{n}(s e)+t(l e)=p\left(p^{n-1} s e+t a\right) \in p S=\Delta(S),
$$

получаем противоречие. Значит, $h=p^{n}$.

Лемма 1. Пусть $S$ - кольцо характеристики $p^{n}$ такое, что $S / p S-$ полутело, где $p, n$ - натуральные числа, $p$ - простое число. Пусть а - элемент кольча $S$, тогда

(1) если существует число $t \in\{0,1, \ldots, n-1\}$ такое, что $p^{t} a=0$, то a $\in p S$;

(2) существуют единственное натуральное число $t \in\{0,1, \ldots, n\}$ и элемент $a_{t} \in(S \backslash p S)$ (не обязательно единственный) такие, что $a=p^{t} a_{t}$; если $a \in p S$, то $t \geqslant 1$.

Доказательство. При $n=1$ лемма очевидна. Пусть далее $n>1$.

Пусть $a \notin p S$. В этом случае $\bar{a} \neq \overline{0}$ и существует элемент $b \in S$ такой, что $\bar{e}=\overline{a b}$. Тогда $a b=e+p s$ для некоторого элемента $s \in S$. При этом

$$
0=\left(p^{t} a\right) b=p^{t} e+p^{t+1} s
$$

и $p^{n-1} e=0$, что противоречит определению характеристики кольца. Следовательно, $a \in p S$.

Если $a=0$, то утверждение очевидно при $t=n$. Пусть $a \neq 0$. Так как $p^{n-1} S \neq 0$, то кольцо $S$ содержит строго убывающую цепочку идеалов

$$
S \triangleright p S \triangleright \ldots \triangleright p^{n-1} S \triangleright p^{n} S=0
$$

и существует единственный параметр $t \in\{0,1, \ldots, n-1\}$ такой, что $a \in\left(p^{t} S \backslash p^{t+1} S\right)$. В таком случае $a=p^{t} a_{t}$, где $a_{t} \in S$, и, более того, $a_{t} \notin p S$, так как иначе $a \in p^{t+1} S$. Предположим, что

$$
a=p^{t} a_{t}=p^{s} a_{s}
$$

где $t \neq s$ и $a_{t}, a_{s} \notin p S$. Если $t<s$, то

$$
p^{n-1} a_{t}=p^{n-1-t} a=p^{n-1+s-t} a_{s}=p^{n} a_{s}=0,
$$

и первая часть леммы показывает, что $a_{t} \in p S$. Аналогично, если $s<t$, то $a_{s} \in p S$. Следовательно, число $t$ определено однозначно. 
Теперь мы можем следующим образом охарактеризовать обобщенное кольцо Галуа $S$ в терминах характеристики кольца и верхнего фактора $S / p S$.

Теорема 1. Конечное кольчо $S$ с единицей е есть обобщенное кольцо Галуа в том и только в том случае, если существуют простое число р и натуральное число п такие, что char $S=p^{n}$ u $S / p S-$ полутело.

Доказательство. Необходимость условий вытекает из предложения 3.

Предположим, что условия теоремы выполнены, и покажем, что $\Delta(S)=p S$. Пусть $a$ и $b$ - два ненулевых элемента кольца $S$ такие, что $a b=0$. Требуется доказать, что $a, b \in p S$. Предположим, что $b$ не принадлежит $p S$ (доказательство для случая $a \notin p S$ аналогично). Поскольку в полутеле $S / p S$ выполняется равенство $\bar{a} \bar{b}=\overline{0}$, получаем, что $\bar{a}=\overline{0}$, то есть $a \in p S$. Следовательно, можно найти натуральное число $t \in\{1, \ldots, n-1\}$ и элемент $a_{t} \in S \backslash p S$ такие, что $a=p^{t} a_{t}$ (см. лемму 1). Поэтому $0=a b=p^{t}\left(a_{t} b\right)$ и $a_{t} b \in p S$ в силу леммы 1). Таким образом, $\overline{0}=\overline{a_{t}} \bar{b}$, откуда $a_{t} \in p S$ или $b \in p S$, что противоречит предположению. Поэтому $b$ принадлежит $p S$, из чего следует, что $\Delta(S) \subseteq p S$. Легко видеть, что $p S \subseteq \Delta(S)$, поскольку $p^{n-1} e(p S)=0$, что и завершает доказательство.

Определение 6. Пусть $D$ - конечное полутело характеристики $p$. Для любого натурального числа $n$ обозначим через $G G R\left(D, p^{n}\right)$ класс всех обобщенных колец Галуа $S$ характеристики $p^{n}$ с верхним фактором $\bar{S}=S / p S$, изоморфным полутелу $D$.

Определение 7. Для обобщенного кольца Галуа $S$ характеристики $p^{n}$ и для любого натурального числа $t \in \mathbf{N}$ обозначим через $\Delta^{t}$ подгрупाу группы $(S,+)$, порожденную всеми произведениями $s \geqslant t$ элементов $\Delta(S)$.

Следующая теорема показывает, что некоторые свойства ассоциативных колец Галуа выполнены также для произвольного обобщенного кольца Галуа.

Теорема 2. Пусть $(S,+, *) \in G G R\left(D, p^{n}\right)$ - обобщенное кольчо Галуа $c$ единичей $e$ такое, что полутело $D$ содержит $q=p^{r}$ элементs. Тогда

(1) множество $S^{*}=S \backslash p S$ замкнуто относительно умножения *u $\left(S^{*}, *\right)-$ лупа с единицей $e$;

(2) решетка односторонних односторонних идеалов кольча $S$ совпадает со строго убывающей цепью двусторонних идеалов

$$
S=\Delta^{0} \triangleright \Delta^{1} \triangleright \ldots \triangleright \Delta^{n-1} \triangleright \Delta^{n}=0,
$$

более того, для любого а $a \in \Delta^{t} \backslash \Delta^{t+1}, t \in\{0,1, \ldots, n-1\}$ имеют место равенства

$$
S * a=a * S=\Delta^{t}=p^{t} S
$$

(3) мощность множества $S$ равна $q^{n}$, и справедливы равенства

$$
\left|S^{*}\right|=q^{n}-q^{n-1}, \quad\left|\Delta^{t}\right|=q^{n-t}, \quad t \in\{0,1, \ldots, n\} .
$$


Доказательство. Докажем утверждение (1). Пусть $a, b \in S^{*}$, тогда $\bar{a}, \bar{b} \neq \overline{0}$. Поскольку $\bar{S}$ - полутело,

$$
\overline{0} \neq \bar{a} * \bar{b}=\overline{a * b}
$$

откуда $a * b \notin p S$. Поэтому множество $S^{*}$ замкнуто относительно умножения $*$. Теперь пусть $a, b, c \in S^{*}$ таковы, что $b \neq c$. Покажем, что тогда $a * b \neq a * c$. Действительно, в противном случае $\bar{a} * \overline{b-c}=\overline{0}$, и так как $\bar{S}$ - полутело, $\overline{b-c}=\overline{0}$. Поэтому $b-c \in p S$ и, по второй части леммы $1, b-c=p^{t} s$ для некоторых $s \notin p S$ и $t \in\{1, \ldots, n\}$. Если $t=n$, то $b=c$, что противоречит предположению. Если $t<n$, то поскольку $0=a *(b-c)=p^{t}(a * s)$, по первой части леммы $1, a * s \in p S$. С другой стороны, множество $S^{*}$ замкнуто относительно $*$, поэтому $a * s \notin p S$. Противоречие доказывает требуемое неравенство $a * b \neq a * c$. В силу конечности множества $S^{*}$, очевидно, что $\left(S^{*}, *\right)$ - лупа с единицей $e$.

Докажем утверждение (2). Легко видеть, что $\Delta^{t}-$ двусторонний идеал кольца $S$ и что $\Delta^{t}=p^{t} S$ для любого натурального числа $t \in\{0,1, \ldots, n\}$. Теперь пусть $I-$ собственный односторонний идеал кольца $S$ (допустим, что это правый идеал). Тогда существует $t \in\{0,1, \ldots, n-1\}$ такое, что $I \subseteq \Delta^{t}$, но $I \nsubseteq \Delta^{t+1}$. Мы покажем, что $I=\Delta^{t}$. По условию найдутся элементы $a \in I, a_{t} \in S^{*}$ такие, что $a=p^{t} a_{t}$. Докажем, что $a_{t} * S=S$. Пусть $b$ - элемент кольца $S$. Существуют $l \in\{0,1, \ldots, n\}$ и $c \in S^{*}$ такие, что $b=p^{l} c$. Следовательно, так как $\left(S^{*}, *\right)-$ лупа, существует такой элемент $h \in S$, что $a_{t} * h=c$. Ясно, что

$$
b=p^{l} c=a_{t} *\left(p^{l} h\right) \in a_{t} * S,
$$

откуда $a_{t} * S=S$ (доказательство равенства $S * a_{t}=S$ аналогично). Следовательно,

$$
I \subseteq \Delta^{t}=p^{t} S=p^{t}\left(a_{t} * S\right)=\left(p^{t} a_{t}\right) * S=a * S \subseteq I,
$$

поскольку $I$ - правый идеал кольца $S$. Из этого вытекает, что $I=\Delta^{t}=p^{t} S$.

Чтобы доказать (3) достаточно показать, что для любого $t \in\{0,1, \ldots, n-1\}$ мощность множества $\Delta^{t} / \Delta^{t+1}$ равна $q$. Очевидно, что $\left(\Delta^{t} / \Delta^{t+1},+\right)$ - абелева группа. Определим отображение

$$
\begin{aligned}
\varphi: S & \rightarrow \Delta^{t} / \Delta^{t+1}, \\
a & \mapsto p^{t} a+\Delta^{t+1} .
\end{aligned}
$$

Отображение $\varphi-$ эпиморфизм групп, причем

$$
\operatorname{ker}(\varphi)=\left\{a \in S \mid p^{t} a \in \Delta^{t+1}=p^{t+1} S\right\}=p S
$$

Следовательно, $(S / p S,+) \cong\left(\Delta^{t} / \Delta^{t+1},+\right)$ и $\Delta^{t} / \Delta^{t+1}$ содержит $q$ элементов.

Мы завершим этот раздел построением обобщенных колец Галуа, верхние факторы которых - полутела, описанные в примерах 1 и 2 (коммутативные полутела Диксона и обобщенные скрученные поля). 
Пример 3. Рассмотрим ассоциативное кольцо Галуа $R=G R\left(q^{n}, p^{n}\right)$ из $q^{n}=\left(p^{e}\right)^{n}$ элементов характеристики $p^{n}$ для некоторого натурального числа $n \in \mathbf{N}$ ( $p$ - нечетное простое число и $e \geqslant 2$ ). Пусть $g \in R-$ такой элемент, что $\bar{g} \in \bar{R}=R / p R=G F(q)$ не является квадратом в $G F(q)\left(\bar{g} \neq a^{2}\right.$ для любого $\left.a \in G F(q)\right)$. Пусть $\sigma$ - нетождественный автоморфизм кольца $S$.

Определим на множестве $S=R \times R$ покоординатное сложение и рассмотрим новую операцию $*$ такую, что

$$
\forall a, b, c, d \in R:(a, b) *(c, d)=(a c+g \sigma(b) \sigma(d), a d+b c)
$$

Тогда $(S,+, *)$ - обобщенное кольцо Галуа из $q^{2 n}$ элементов, характеристики $p^{n}$, верхний фактор которого - коммутативное полутело Диксона. Этот факт непосредственно следует из примера 1 и теоремы 1.

Нетрудно проверить также, что центр кольца $S$ есть

$$
Z(S)=\{(a, 0): a \in R\}=G R\left(q^{n}, p^{n}\right)
$$

Таким же способом из примера 2 получается следующий пример.

Пример 4. Рассмотрим ассоциативное кольцо Галуа $R=G R\left(q^{n}, p^{n}\right)$ из $q^{n}$ элементов, $q=p^{c}>2$, характеристики $p^{n}$ для некоторого натурального числа $n$. Пусть $S=G R\left(q^{d n}, p^{n}\right)$ - ассоциативное расширение Галуа кольца Галуа $R$ степени $d \geqslant 3$ (см. [12]) и $c$ - такой элемент кольца $S$, что порядок элемента $\bar{c} \in \bar{S}=S / p S=G F\left(q^{d}\right)$ не делит $\left(q^{d}-1\right) /(q-1)$. Пусть $\sigma \neq i d$ and $\tau \neq i d-$ два различных автоморфизма кольца $S$ такие, что

$$
R=\{a \in S \mid \sigma(a)=a=\tau(a)\}
$$

Определим новое произведение элементов множества $S$ следующим образом:

$$
\forall x, y \in S: x \cdot y=x y-c \tau(x) \sigma(y)
$$

Отображения $R_{1}$ и $L_{1}$ модуля $S$ на $S$, заданные формулами

$$
\forall x \in R: \quad R_{1}(x)=x \cdot 1=x-c \tau(x), \quad L_{1}(x)=1 \cdot x=x-c \sigma(x),
$$

биективны. Следовательно, можно рассмотреть обратные отображения $R_{1}^{-1}$ и $L_{1}^{-1}$. Определим еще одно произведение элементов множества $S$ следующим образом:

$$
\forall x, y \in S: x * y=\left(R_{1}^{-1}(x)\right) \cdot\left(L_{1}^{-1}(y)\right)
$$

Тогда $(S,+, *)$ - обобщенное кольцо Галуа характеристики $p^{n}$ и его верхний фактор обобщенное скрученное поле. 


\section{4. Верхне-ассоциативные обобщенные кольца Галуа}

Этот раздел посвящен исследованию обобщенных колец Галуа $S$, верхний фактор $\bar{S}=S / p S$ которых является конечным полем. Естественно задать вопрос, следует ли из ассоциативности верхнего фактора ассоциативность самого кольца. Как будет показано, ответ на этот вопрос - отрицательный: существуют неассоциативные обобщенные кольца Галуа с конечным полем в качестве верхнего фактора. Однако, как мы увидим, более слабое условие ассоциативности (ассоциативность степеней) оказывается достаточным, чтобы поднимать ассоциативность с верхнего фактора на все кольцо.

Определение 8. Пусть $S$ - обобщенное кольцо Галуа. Назовем $S$ верхне-ассоциативным обобщенным кольцом Галуа, если его верхний фактор $\bar{S}=S / p S$ - поле, то есть $S \in G G R\left(G F(q), p^{n}\right)$ для некоторого $q=p^{r}$.

Лемма 2. Пусть $S$ - обобщенное кольцо Галуа (не обязательно верхне-ассочиативное), $R$ - подкольчо чентра кольча $S$, являющееся ассочиативным кольчом Галуа, $R=G R\left(q^{n}, p^{n}\right)$, $q=p^{c}$. Тогда $\bar{S}=S / p S-$ векторное пространство над $\bar{R}=R / p R$ u $S$ - свободный модуль ранга $d=\operatorname{dim}_{\bar{R}} \bar{S}$ над $R$. Более того, произвольное множество $\left\{x_{1}, \ldots, x_{d}\right\}$ из $d$ элементов кольча $S$ является $R$-базисом $S$ тогда и только тогда, когда $\left\{\overline{x_{1}}, \ldots, \overline{x_{d}}\right\}$ является $\bar{R}$-базисом пространства $\bar{S}$.

Доказательство. Лемма легко выводится из леммы Накаямы [5]. Именно, пусть $B$ $R$-подмодуль в $S$, порожденный $\left\{x_{1}, \ldots, x_{d}\right\}$. Если $\left\{\overline{x_{1}}, \ldots, \overline{x_{d}}\right\}$ является $\bar{R}$-базисом пространства $\bar{S}$, то

$$
S=B+p S=B+(p R) S .
$$

Поскольку $p R$ - нильрадикал кольца $R$, применяя лемму Накаямы, получаем, что $S=B$, то есть множество $\left\{x_{1}, \ldots, x_{d}\right\}$ порождает $R$-модуль $S$. Остается показать, что $\left\{x_{1}, \ldots, x_{d}\right\}$ является $R$-базисом модуля $S$. Достаточно заметить, что число различных $R$-линейных комбинаций элементов $\left\{x_{1}, \ldots, x_{d}\right\}$ не превосходит $\left(q^{n}\right)^{d}$, а последнее равно числу элементов кольца $S$ (теорема 2). Так как множество $\left\{x_{1}, \ldots, x_{d}\right\}$ порождает модуль $S$, все $R$-линейные комбинации элементов $\left\{x_{1}, \ldots, x_{d}\right\}$ должны быть различными. Следовательно, система $x_{1}, \ldots, x_{d}$ линейно независима над $R$ и образует $R$-базис (свободного) $R$-модуля $S$ (ранга $d$ ).

Предложение 4. Пусть $S \in G G R\left(G F(q), p^{n}\right)$ - верхне-ассочиативное обобщенное кольчо Галуа характеристики $p^{n}\left(q=p^{c}\right)$. Если $c \leqslant 2$, то $S$ - ассочиативное кольцо Галуа.

Доказательство. Рассмотрим подкольцо $R$, порожденное единицей $e$. Тогда $R$ изоморфно $Z_{p^{n}}=G R\left(p^{n}, p^{n}\right)$ и содержится в центре кольца $S$. Следовательно, $S$ - свободный модуль над $R$, и так как $c \leqslant 2$, ранг этого модуля не превосходит 2 . Если этот ранг равен 1, то ассоциативность кольца $S$ очевидна. Если же этот ранг равен 2 , можно выбрать такой элемент $a \in S$, что множество $\{e, a\}$ является $R$-базисом модуля $S$, следовательно, кольцо $S$ оказывается ассоциативным.

Для любого $c \geqslant 3$ существуют неассоциативные обобщенные кольца Галуа характеристики $p^{n}$ (необходимо большей, чем $p$ ) с конечным полем из $p^{c}$ элементов в качестве верхнего фактора. 
Пример 5. Пусть $S=G R\left(p^{c n}, p^{n}\right)$ - ассоциативное кольцо Галуа характеристики $p^{n}>p$, верхний фактор которого - конечное поле $D=G F\left(p^{c}\right)$. Пусть $t_{1}, t_{2}$ - такие эндоморфизмы групाт $(S,+)$, что $t_{1}(e)=t_{2}(e)=0$. Определим новую операцию $*$ на $S$ следующим образом:

$$
\forall x, y \in S: x * y=x y+p t_{1}(x) t_{2}(y)
$$

Несложно проверить, что в таком случае $(S,+, *)$ - обобщенное кольцо Галуа характеристики $p^{n}$ с верхним фактором $D$. Более того, при $c \geqslant 3$ можно выбрать $t_{1}, t_{2}$ так, что операция * неассоциативна. Например, если $\sigma-$ образующий элемент циклической группы $\operatorname{Aut}(S)$ (см. [14]), можно взять $t_{1}$ и $t_{2}$, определяемые следующим образом:

$$
\forall x \in S: t_{1}(x)=\sigma(x)-x, t_{2}(x)=\sigma^{2}(x)-x .
$$

В этом случае $(S \backslash p S, *)$ даже не является группоидом с ассоциативными степенями. Тем более $(S,+, *)$ - неассоциативное обобщенное кольцо, но его верхний фактор $\bar{S}-$ ассоциативное конечное поле $D$.

Таким образом, ассоциативность обобщенного кольца Галуа $S$ не может быть выведена из ассоциативности его верхнего фактора $\bar{S}$. Покажем теперь, что любое верхнеассоциативное обобщенное кольцо Галуа с ассоциативными степенями - ассоциативное кольцо Галуа.

Лемма 3. Пусть $S \in G G R\left(G F(q), p^{n}\right)$ - верхне-ассочиативное обобщенное кольчо Галуа, $q=p^{c}$, характеристики $p^{n}, a-$ элемент множества $S \backslash p S$. Если степени а ассочиативны (то есть подкольчо, порожденное элементом а, ассочиативно), то множество $A$ этих степеней является (относительно операчии произведения в $S$ ) конечной чиклической группой, порядок которой делит число $(q-1) p^{n-1}$.

Доказательство. Очевидно, что множество $A$ является полугруппой, так как оно замкнуто относительно умножения и ассоциативно. Покажем, что единица $e$ кольца $S$ принадлежит $A$.

Для любого элемента $b \in S$ обозначим через $b^{t}$ правую главную степень $t$ элемента $b$ :

$$
b^{1}=b, \quad b^{t}=b^{t-1} \cdot b .
$$

Так как $a \notin p S, \bar{a} \in G F(q)^{*}$ и, следовательно, $\overline{a^{q-1}}=\bar{e}$. Значит, существует такой элемент $h_{1} \in S$, что $a^{q-1}=e+p h_{1}$. Поскольку $e$ и $p h_{1}$ коммутируют, справедливо равенство

$$
\left(e+p h_{1}\right)^{p}=e+\left(\begin{array}{l}
p \\
1
\end{array}\right) p h_{1}+\ldots+\left(\begin{array}{c}
p \\
p-1
\end{array}\right) p^{p-1} h_{1}^{p-1}+p^{p} h_{1}^{p}=e+p^{2} h_{2}
$$

для некоторого $h_{2} \in S$. Используя ассоциативность степеней элемента $a$, получаем, что

$$
\begin{aligned}
\left(e+p h_{1}\right)^{p^{2}} & =\left(\left(e+p h_{1}\right)^{p}\right)^{p}=\left(e+p^{2} h_{2}\right)^{p} \\
& =e+\left(\begin{array}{c}
p \\
1
\end{array}\right) p^{2} h_{2}+\ldots+\left(\begin{array}{c}
p \\
p-1
\end{array}\right)\left(p^{2}\right)^{p-1}\left(h_{2}\right)^{p-1}+\left(p^{2}\right)^{p}\left(h_{2}\right)^{p}=e+p^{3} h_{3}
\end{aligned}
$$

для некоторого $h_{3} \in S$. 
Этот процесс можно продолжить, пока не получим равенство

$$
\left(e+p h_{1}\right)^{p^{n-1}}=e+p^{n} h_{n}=e,
$$

выполненное для некоторого $h_{n} \in S$. Но тогда

$$
\left(a^{q-1}\right)^{p^{n-1}}=\left(e+p h_{1}\right)^{p^{n-1}}=e .
$$

Таким образом, $e \in A$.

Наконец, очевидно, что $b=a^{(q-1) p^{n-1}-1}$ - элемент множества $A$, и $a b=b a=e$.

Заметим также, что мультипликативный порядок элемента $a$ делит число $(q-1) p^{n-1}$. Следовательно, $A$ - группа, она является циклической и ее порядок делит число $(q-1) p^{n-1}$.

Предложение 5. Пусть $S \in G G R\left(G F(q), p^{n}\right)$ - верхне-ассочиативное $G G R, q=p^{c}$, содержащее такой элемент $a \in S \backslash p S$ с ассочиативными степенями, что $\bar{a} \in \bar{S}$ порождает мультипликативную группу $\bar{S}^{*}$. Тогда $S$ - ассочиативное и коммутативное кольцо, то есть $S$ - ассочиативное кольчо Галуа.

Доказательство. Элемент $a$ порождает циклическую группу, порядок которой делит число $(q-1) p^{n-1}$. Порядок элемента $\bar{a}$ равен $q-1$, так как этот элемент порождает мультипликативную группу $G F(q)^{*}$, следовательно, $q-1$ делит порядок элемента $a$. Поэтому порядок $a$ равен $(q-1) p^{s}$ для некоторого $s \in\{0,1, \ldots, n-1\}$. Значит, элемент $\theta=a^{p^{s}}$ имеет мультипликативный порядок $q-1$ и выполнено равенство

$$
G F(q)=\bar{S}=\left\{0, \bar{\theta}, \overline{\theta^{2}}, \ldots, \overline{\theta^{q-1}}\right\} .
$$

Пусть $\Gamma(S)$ - множество $\left\{0, \theta, \theta^{2}, \ldots, \theta^{q-1}\right\} \subseteq S$. Легко показать, что для любого элемента $b \in S$ существует единственный набор элементов $\left\{\beta_{0}, \beta_{1}, \ldots, \beta_{n-1}\right\} \subseteq \Gamma(S)$ такой, что

$$
b=\beta_{0}+p \beta_{1}+\ldots+p^{n-1} \beta_{n-1} .
$$

Следовательно, для кольца $S$ справедливо разложение

$$
S=\Gamma(S)+p \Gamma(S)+\ldots+p^{n-1} \Gamma(S) .
$$

С другой стороны, подкольцо кольца $S$, порожденное множеством $\Gamma(S)$, ассоциативно и коммутативно. Но, как мы только что видели, $\Gamma(S)$ порождает $S$, так что $S$ - ассоциативное и коммутативное кольцо.

Следствие 1. Любое верхне-ассочиативное обобщенное кольчо Галуа с ассочиативньми степенями - ассочиативное кольчо Галуа.

Исследование произвольных обобщенных колец Галуа с ассоциативными степенями (необязательно верхне-ассоциативных) почти сводится к исследованию верхне-ассоциативных обобщенных колец Галуа с ассоциативными степенями. Следующая известная теорема, доказанная А. А. Албертом [1] в 1952 г. и передоказанная К. Маккриммоном [10] в 1966 г., утверждает, что почти все конечные полутела с ассоциативными степенями суть конечные поля.

Теорема 3. Пусть D - конечное полутело с ассочиативными степенями, характеристика которого отлична от 2 , причем его иентр $Z(D)$ - конечное поле, содержачее не меньше 7 элементов. Тогда $D$ - конечное поле. 
Из теоремы 3 вытекает следующее утверждение.

Следствие 2. Пусть $S$ - обобщенное кольцо Галуа с ассочиативными степенями нечетной характеристики, верхний фактор которого $\bar{S}=S / p S-$ полутело с иентром, содержащим не менее 7 элементов. Тогда $S$ - ассочиативное кольчо Галуа.

Таким образом, почти все обобщенные кольца Галуа с ассоциативными степенями ассоциативны.

\section{5. Теорема существования}

В последнем разделе мы докажем теорему существования для обобщенного кольца Галуа с заданными характеристикой и верхним фактором. Будет показано, что для любого заданного конечного полутела $D$ характеристики $p$ и любого натурального числа $n$ существует обобщенное кольцо Галуа $S$ характеристики $p^{n}$ с верхним фактором $\bar{S}=S / p S \cong D$, то есть класс $G G R\left(D, p^{n}\right)$ не пуст. Более того, мы докажем существование обобщенного кольца Галуа $S$, удовлетворяющего этим условиям, центр которого - ассоџиативное кольцо Галуа, проектирующееся на центр $\bar{S}$ при естественном эпиморфизме $S \rightarrow \bar{S}$.

Предложение 6. Пусть $S$ - обобщенное кольцо Галуа характеристики $p^{n}$. Тогда

(1) подкольчо $R$ кольца $S$ с единичей е есть обобщенное кольчо Галуа тогда и только тогда, когда $p R=p S \cap R$;

(2) если центр $Z(S)$ кольца $S$ является (ассочиативным) кольцом Галуа, то верхний фактор $\overline{Z(S)}=Z(S) / p Z(S)$ изоморфен подкольчу кольча $Z(\bar{S})$.

Доказательство. Докажем (1). Допустим, что $R$ - обобщенное кольцо Галуа. Очевидно, что $p R \subseteq p S \cap R$. Рассмотрим элемент $a \in p S \cap R$. Если $a \notin p R$, то, поскольку $R \backslash p R$ является лупой (теорема 2), существует такой элемент $b \in R \backslash p R$, что $a * b=e$. Но тогда

$$
p^{n-1} e=p^{n-1}(a * b)=\left(p^{n-1} a\right) * b=0,
$$

так как $a \in p S$, что противоречит предположению о характеристике кольца $S$.

Обратно, пусть $p R=p S \cap R$. Ясно, что $R$ - конечное кольцо с единицей $e$ характеристики $p^{n}$. Рассмотрим канонический эпиморфизм $\pi: S \rightarrow \bar{S}$ и заметим, что его ограничение $\varphi$ на подкольцо $R$ индуцирует изоморфизм

$$
R / \operatorname{ker} \varphi=R /(p S \cap R)=R / p R \cong \operatorname{Im} \varphi \leqslant \bar{S} \text {. }
$$

Таким образом, $R / p R$ - подкольцо с единицей конечного полутела, следовательно, оно само - полутело. Следовательно, согласно теореме $1, R$ - обобщенное кольцо Галуа.

Докажем (2). Пусть $\pi: S \rightarrow \bar{S}-$ канонический эпиморфизм. Очевидно, что $\pi(Z(S)) \subseteq Z(\bar{S})$ и $\pi$ индуцирует гомоморфизм $\varphi: Z(S) \rightarrow Z(\bar{S})$. Ввиду первого утверждения

$$
\operatorname{ker} \varphi=Z(S) \cap p S=p Z(S) \text {, }
$$

откуда

$$
\overline{Z(S)}=Z(S) / p Z(S) \cong \varphi(Z(S)) \subseteq Z(\bar{S})
$$


Определение 9. Пусть $S$ - обобщенное кольцо Галуа характеристики $p^{n}$. Если имеет место изоморфизм

$$
\overline{Z(S)}=Z(S) / p Z(S) \cong Z(\bar{S}),
$$

то будем говорить, что $S$ - поднятие полутела $\bar{S}$ характеристики $p^{n}$.

Заметим, что если $S$ - обобщенное кольцо Галуа характеристики $p^{n}$ и его верхний фактор $\bar{S}$ - полутело с центром $P=G F(q)$, то $S$ - поднятие $\bar{S}$ характеристики $p^{n}$ тогда и только тогда, когда центр $S$ изоморфен $G R\left(q^{n}, p^{n}\right)$ (так как ассоциативное кольцо Галуа полностью определяется его мощностью и характеристикой).

Теорема 4. Для любого полутела D характеристики р и для любого натурального числа $n$ сушествует поднятие $S$ полутела $D$ характеристики $p^{n}$.

Доказательство. Для доказательства теоремы сначала введем некоторые обозначения. Рассмотрим полутело $D$ как векторное пространство над его центром $P=G F(q), q=p^{c}$. Пусть $d$ - его размерность. Зафиксируем базис $D_{P}$

$$
x=\left(x_{1}=\varepsilon, x_{2}, \ldots, x_{d}\right),
$$

где $\varepsilon$ - единица полутела $D$. Для любого элемента $\alpha \in D$ существует единственный вектор-столбец координат $\alpha_{x} \in P^{(d)}$ с элементами из $P$. Тогда элемент $\alpha \in D$ представляется в виде произведения

$$
\alpha=x \alpha_{x}=\alpha_{x}^{t} x^{t} .
$$

Более того, существует такая квадратная матрица размера $d \times d$ над $D$

$$
g=\left(g_{i j}\right)_{i, j=1, \ldots, d}, \quad g_{i j}=x_{i} x_{j}, \quad i, j=1, \ldots, d,
$$

что произведение в $D$ задается следующим образом:

$$
\forall \alpha, \beta \in D: \alpha \beta=\alpha_{x}^{t} g \beta_{x}
$$

Заметим, что первая строка матрицы $g$ совпадает с вектором $x$ и что ее первый столбец равен $x^{t}$, так как $x_{1}=\varepsilon-$ единица полутела.

C другой стороны, мы можем определить новую операцию - на множестве $D$ :

$$
\forall \alpha, \beta \in P: \alpha \cdot \beta=\alpha \beta
$$

Тогда кольцо $(D,+, \cdot)$ есть конечное поле $G F\left(q^{d}\right)$ - расширение поля $P$ степени $d$.

Построим теперь поднятие полутела $D$ характеристики $p^{n}$ для любого натурального числа $n$.

Пусть $R=G R\left(q^{n}, p^{n}\right)$ - ассоциативное кольцо Галуа мощности $q^{n}$ и характеристики $p^{n}$. Пусть $S=G R\left(q^{d n}, p^{n}\right)$ - расширение Галуа степени $d$ кольца $R$ (см. $\left.[15,12]\right)$. Тогда $S-$ свободный бимодуль ранга $d$ над $R$. Более того,

$$
\bar{S}=G F\left(q^{d}\right) \cong(D,+, \cdot) .
$$

Зафиксируем базис модуля $S_{R}$

$$
y=\left(y_{1}=e, y_{2}, \ldots, y_{d}\right),
$$

5 Дискретная математика, т.14 №4 
где $e$ - единица кольца $S$ и $\overline{y_{i}}=x_{i}$ для всех $i \in\{2, \ldots, d\}$. Обозначим через $a_{y}$ векторстолбец координат (в $R$ ) элемента $a \in S$ относительно базиса $y$. Тогда

$$
a=y a_{y}=a_{y}^{t} y^{t} .
$$

Зафиксируем матрицу $G$ размера $d \times d$ над модулем $S$

$$
G=\left(G_{i j}\right)_{i, j=1, \ldots, d}
$$

каждый элемент $G_{i j}$ которой удовлетворяет условию

$$
\bar{G}_{i j}=g_{i j}, \quad i, j=1, \ldots, d,
$$

причем первая строка матрицы $G$ совпадает с вектором $y$, а ее первый столбец - с вектором $y^{t}$.

Определим новую операцию $*$ на $S$ следующим образом:

$$
\forall a, b \in S: a * b=a_{y}^{t} G b_{y} .
$$

Назовем матрицу $G=G(y)$ матрицей Грама операции * относительно базиса $y$. Покажем теперь, что $(S,+, *)$ - поднятие полутела $D$ характеристики $p^{n}$. Очевидно, что $S-$ конечное кольцо с единицей $y_{1}=e$. Определим следующее отображение кольца $(S,+, *)$ на кольцо $D$ :

$$
\begin{aligned}
\varphi: S & \rightarrow D, \\
a & \mapsto \varphi(a)=x \bar{a}_{y}
\end{aligned}
$$

(для любого элемента $a \in S$ с координатами $a_{y}=\left(\alpha_{1}, \ldots, \alpha_{d}\right)^{t}$ относительно базиса $y, \bar{a}_{y}$ обозначает вектор $\left.\left(\overline{\alpha_{1}}, \ldots, \overline{\alpha_{d}}\right)^{t} \in P^{(d)}\right)$. Легко проверяется, что $\varphi$ - эпиморфизм колец с ядром $p S$. Следовательно, из теоремы 1 вытекает, что $(S,+, *)-$ обобщенное кольцо Галуа характеристики $p^{n}$ с верхним фактором $S / p S$, изоморфным $D$.

Осталось только доказать, что $S$ - поднятие $D$. Покажем, что центр кольца $(S,+, *)$ совпадает с множеством $Z=R y_{1}=\operatorname{Re}$ и $(Z,+, *)=G R\left(q^{n}, p^{n}\right)$. Очевидно, что $\lambda y_{1}-$ центральный элемент кольца $(S,+, *)$ для любого $\lambda \in R$. Обратно, пусть $a$ - элемент центра кольца $(S,+, *)$. Можно выразить $a$ в виде

$$
a=\lambda y_{1}+z,
$$

где $\lambda \in R$ и $z \in R y_{2}+\ldots+R y_{d}$. Покажем, что $a \in Z$, то есть, что $z=0$. Поскольку $a$ - центральный элемент кольца $(S,+, *)$, элемент $\bar{a} \in \bar{S} \cong D$ принадлежит центру $Z(D)=P=P x_{1}$ полутела $D$. Следовательно, $\bar{z}=\overline{0}$. Допустим, что $z \neq 0$. В этом случае в силу леммы 1) можно найти такие число $t \in\{1, \ldots, n-1\}$ и элемент $z_{t} \in S^{*}$, что $z=p^{t} z_{t}$. Если $\overline{z_{t}}$ принадлежит $Z(D)$, то

$$
z_{t}=\mu y_{1}+w
$$

где $\mu \in R, w \in R y_{2}+\ldots+R y_{d}$ и $\bar{w}=0$. Так как $z_{t} \notin p S$,

$$
0 \neq \overline{z_{t}}=\overline{\mu y_{1}+w}=\bar{\mu} x_{1}+\bar{w}=\bar{\mu} x_{1} .
$$

Следовательно, $\mu \notin p R$. Но

$$
z=p^{t} z_{t}=p^{t}\left(\mu y_{1}+w\right)=\left(p^{t} \mu\right) y_{1}+p^{t} w
$$


принадлежит $R y_{2}+\ldots+R y_{d}$. Поэтому $\left(p^{t} \mu\right) y_{1}=0$ и лемма 1 утверждает, что $\mu \in p R$, что противоречит показанному выше. Следовательно, $\overline{z_{t}}$ не принадлежит $Z(D)$ и можно найти элементы $r, s \in S$ и отображение $f: S \times S \times S \rightarrow S$ одного из следующих четырех видов:

$$
\begin{array}{ll}
f\left(z_{t}, r, s\right)=\left(z_{t} r\right) s-z_{t}(r s), & f\left(z_{t}, r, s\right)=\left(r z_{t}\right) s-r\left(z_{t} s\right), \\
f\left(z_{t}, r, s\right)=(r s) z_{t}-r\left(s z_{t}\right), & f\left(z_{t}, r, s\right)=z_{t} r-r z_{t},
\end{array}
$$

то есть (ассоциатор или коммутатор) отображение такое, что индуцированное отображение $\bar{f}: D \times D \times D \rightarrow D$ (также ассоциатор или коммутатор) удовлетворяет условию

$$
\bar{f}\left(\overline{z_{t}}, \bar{r}, \bar{s}\right) \neq \overline{0} .
$$

Заметим, что $a$ - элемент центра кольца $S$, поэтому

$$
0=f(a, r, s)=f\left(\lambda y_{1}+p^{t} z_{t}, r, s\right)=f\left(\lambda y_{1}, r, s\right)+p^{t} f\left(z_{t}, r, s\right)=p^{t} f\left(z_{t}, r, s\right) .
$$

Но из леммы 1 следует, что $f\left(z_{t}, y, s\right) \in p S$, то есть $\bar{f}\left(\overline{z_{t}}, \bar{r}, \bar{s}\right)=\overline{0}$, что противоречит предположению. Таким образом, $z=0, a \in Z$ и равенство

$$
Z((S,+, *))=Z \cong R=G R\left(q^{n}, p^{n}\right)
$$

доказано. Следовательно, $S$ - поднятие полутела $D$ характеристики $p^{n}$.

Предыдущая конструкщия показывает, что, вообще говоря, может существовать несколько неизоморфных поднятий фиксированного неассоциативного полутела $D$, поскольку для фиксированного верхнего фактора $D$ матрица Грама $G$, удовлетворяющая условию (14), может быть выбрана различными способами.

Для примера построим два неизоморфных поднятия коммутативного полутела Диксона одной и той же характеристики.

Пример 6. Рассмотрим обобщенное кольцо Галуа $S$ из примера 3 . Легко показать, что оно является поднятием коммутативного полутела Диксона характеристики $p^{n}$ из примера 1 . Более того, кольцо $S$ коммутативно.

Теперь возьмем число $n \geqslant 2$ и определим на $S$ новое умножение $\odot$. Пусть $t_{1}, t_{2}-$ два различных эндоморфизма группы $(R,+)$ такие, что $t_{1}(x)=t_{2}(x)=0$, если $\sigma(x)=x$, где $\sigma$ - автоморфизм кольца $R$, описанный в примере 3. Определим $\odot$ следующей формулой:

$$
\forall a, b, c, d \in R:(a, b) \odot(c, d)=\left(a c+g \sigma(b) \sigma(d)+p t_{1}(a) t_{2}(c), a d+b c\right) .
$$

Тогда $(S,+, \odot)$ также является поднятием коммутативного полутела Диксона характеристики $p^{n}$. Но операция $\odot$ некоммутативна, следовательно, $(S,+, *)$ и $(S,+, \odot)$ суть неизоморфные поднятия.

Описание поднятий заданного полутела с точностью до изоморфизма остаётся открытой проблемой. Было бы интересно найти число классов неизоморофных обобщенных колец Галуа с заданным верхним фактором (или поднятий заданного полутела), имеющих заданную характеристику.

С другой стороны, известно [12], что ассоциативное кольцо Галуа однозначно, с точностью до изоморфизма, определяется своими верхним фактором и характеристикой. Поскольку в неассоциативном случае это уже не так, представляется естественным поставить следующий вопрос: найти дополнительные условия на обобщенное кольцо Галуа, гарантирующие, что обобщенное кольцо Галуа (или поднятие) однозначно, с точностью до изоморфизма, определяется своими верхним фактором и характеристикой. 


\section{Список литературы}

1. Albert A. A., On nonassociative division algebras. Trans. Amer. Math. Soc. (1952) 72, 296-309.

2. Albert A. A., Generalized twisted fields. Pacific J. Math. (1961) 11 (1961), 1-8.

3. Cordero M., Wene G. P., A survey of semifields. Discrete Math. (1999) 208/209, 125-137.

4. Dickson L. E., On commutative linear algebras in which division is always uniquely possible. Trans. Amer. Math. Soc. (1906) 7, 514-522.

5. Hungerford T. W., Algebra. Springer, Berlin, 1974.

6. Janusz G. J., Separable algebras over commutative rings. Trans. Amer. Math. Soc. (1996) 122, 461-478.

7. Knuth D. E., Finite semifields and projective planes. J. Algebra (1965) 2, 182-217.

8. Krull W., Algebraische Theorie der Ringe. II. Math. Ann. (1924) 91, 1-46.

9. Кузьмин А. С., Нечаев А. А., Линейные рекуррентные последовательности над колыцом Галуа. Алгебра и логика (1995) 34, №2, 1-17.

10. McCrimmon K., Finite power-associative division rings. Proc. American Math. Soc. (1966) 17, 1173-1177.

11. McDonald B. R., Finite rings with identity. Wiley, New York, 1969.

12. Нечаев А. А, Конечные кольца главных идеалов. Матем. сб. (1973) 91, №3, 350-366.

13. Нечаев А. А., Базис обобщенных тождеств конечного коммутативного кольца главных идеалов. Алгебра и логика (1979) 18, №2, 186-194.

14. Нечаев А. А., Код Кердока в циклической форме. Дискретная математика (1989) 1, №4, 123139.

15. Raghavendran R., Finite associative rings. Compos. Math. (1969) 21, №2, 195-219.

16. Schafer R. D., An introduction to nonassociative algebras. Academic Press, New York, 1966.

Статья поступила 28.10.2002. 